Advances in Intelligent Systems and Computing 821

Sebastiano Bagnara

Riccardo Tartaglia · Sara Albolino

Thomas Alexander · Yushi Fujita

\title{
Editors
}

Proceedings of the

20th Congress of

the International

Ergonomics

Association (IEA 2018)

Volume IV: Organizational Design and Management (ODAM), Professional Affairs, Forensic

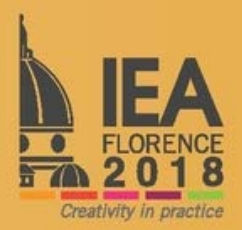

iㅡㄹ Springer 
Sebastiano Bagnara · Riccardo Tartaglia · Sara Albolino - Thomas Alexander - Yushi Fujita Editors

\section{Proceedings of the 20th Congress of the International Ergonomics Association (IEA 2018)}

Volume IV: Organizational Design and Management (ODAM), Professional Affairs, Forensic

This book presents the proceedings of the 2oth Congress of the International Ergonomics Association (IEA 2018), held on August 26-30, 2018, in Florence, Italy. By highlighting the latest theories and models, as well as cutting-edge technologies and applications, and by combining findings from a range of disciplines including engineering, design, robotics, healthcare, management, computer science, human biology and behavioral science, it provides researchers and practitioners alike with a comprehensive, timely guide on human factors and ergonomics. It also offers an excellent source of innovative ideas to stimulate future discussions and developments aimed at applying knowledge and techniques to optimize system performance, while at the same time promoting the health, safety and wellbeing of individuals. The proceedings include papers from researchers and practitioners, scientists and physicians, institutional leaders, managers and policy makers that contribute to constructing the Human Factors and Ergonomics approach across a variety of methodologies, domains and productive sectors.

This volume includes papers addressing Organizational Design and Management.
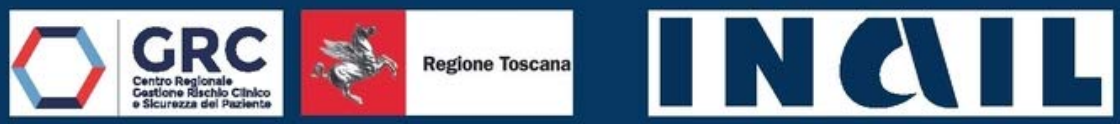

Engineering

ISSN 2194-5357

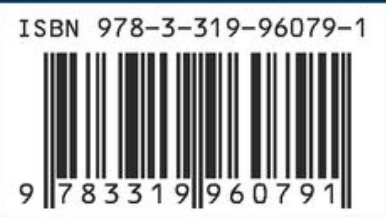

springer.com 


\title{
The Emotion of Light Instrument for Wellness
}

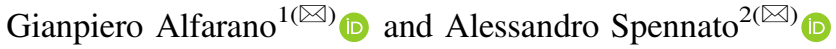 \\ ${ }^{1}$ Department of Architecture, University of Florence, Florence, Italy \\ gianpiero.alfarano@unifi.it \\ 2 Department of Architecture and Design, University of Genoa, Genoa, Italy \\ alessandro.spennato@unifi.it
}

\begin{abstract}
If objects have the ability to talk, if environments have the chance to get around this, it is definitely due to light. If this is spontaneously accepted less spontaneously we accept that objects and environments have their own autonomous experience with whoever uses them.

Yet in them there are phenomena that do not depend on the wishes of the user, but rather express conditions that suggest moods, purely emotional perceptions.

The logic that wants the isometry of the form to determine functions or uses is often a logic of perceptual suggestions, relational relationships between light and shadow. Light not only illuminates but characterizes shapes and spaces. Not only does it allow perception, but it is through it that emotional relationships arise. Objects, spaces through light modulation, chromatic declination, and tonal intensity, offer varying dimensions of fruition.

It is an example that if physical space is always equal to itself in time, luminous space can change and transform perceptual conditions by cognitively altering and even suggesting the psychological relationship of the use of things.

The new dimension of designing light overtake, with the support of increasingly sophisticated implementation tools, the commitment to make a correct contribution to lumens by expanding the lighting design to a strongly cultural trajectory: educating well-being by educating the perception of light.
\end{abstract}

Keywords: Lighting design $\cdot$ Perception $\cdot$ Comfort $\cdot$ Culture of light Sensoriality

If objects have the ability to talk, if environments have the chance to get around this, it is definitely due to light. If this is spontaneously accepted less spontaneously we accept that objects and environments have their own autonomous experience with whoever uses them.

Yet in them there are phenomena that do not depend on the wishes of the user, but rather express conditions that suggest moods, purely emotional perceptions.

The logic that wants the isometry of the form to determine functions or uses is often a logic of perceptual suggestions, relational relationships between light and shadow. Light not only illuminates but characterizes shapes and spaces. Not only does it allow perception, but it is through it that emotional relationships arise. Objects, spaces through light modulation, chromatic declination, and tonal intensity, offer varying dimensions of fruition.

It is an example that if physical space is always equal to itself in time, luminous space can change and transform perceptual conditions by cognitively altering and even suggesting the psychological relationship of the use of things. 
Designing the light today also means refining the approaches to the discipline by adopting training pathways that can interact in close co-operation both with the design of physical parameters and with the intangible content project.

To this is added education to perception as a cultural structure. As Arnheim has already largely argued, perception requires time and in this need to be educated to be aware of it.

According to Arnheim, our perception, in the general sense, is not differentiated beyond what is necessary. That is to say that the concepts are so generic as it is allowed by applying them to understand what you are observing.

To perceive an object as unchangeable means to move it to the highest possible level of generality. In the physical world, however, the modifications exist. The control and design of this variability condition greatly contributes to the comfort of fruition to be more in line with what we actually see, otherwise everything will remain motionless enough to suggest that variations either do not exist or do not matter, thus renouncing emotional sensory.

On this increased awareness, accelerated by the evolution of technological possibilities, new aspects of the light project are being developed today. Now skilled in scientific hardship, the lighting designer needs to draw on the sweet sensuality of light as a research tool for visual comfort before even as a method of rationalizing perceptual cognition.

The tools that the culture of light design has already refined, converge largely towards the acquisition of the founding principle that light can be declined as an emotional value. The light passes from a necessary condition to see a perceptive element that arouses moods. The light used as an expressive language translates into symbolic value. The cultural direction of perception greatly orients the suggestible capacities of communication. The more the light is "hot" the more it works as an attractor. It becomes "fire" around which to collect.

Emotionally it generates a symbolic perception, an imaginary that supplies security and comfort.

The fact that light is a symbolic emotion tells us that it is not possible to find the same path in the attribution of symbolic value both for "warm" and "cold" light.

If the warm light associates an emotional imagery that welcomes and warms, for the cold light we are in a cultural evolutionary stage that becomes commonplace to associate the colors that go towards the blue at low temperatures.

Today the symbolic value of this graduality of light is associated with new technologies. It combines with the most advanced performance of innovative materials.

In this new perceptive perspective, photoluminescence is included. The signs of change can be appreciated, with various perceptive fluctuations, precisely in the ability of the new generations of photoluminescent pigments to be a vector of cultural orientation (see Fig. 1).

To appreciate the considerable shift in the cognitive parameter to which we are subjecting, we must first reaffirm a basic theoretical concept that has accelerated this ongoing process. There is no light but there is light in all its forms. It seems an axiomatic, purely conceptual postulate, but it has been the most significant stimulus that in recent decades has given awareness to the use of light as an emotional component. 

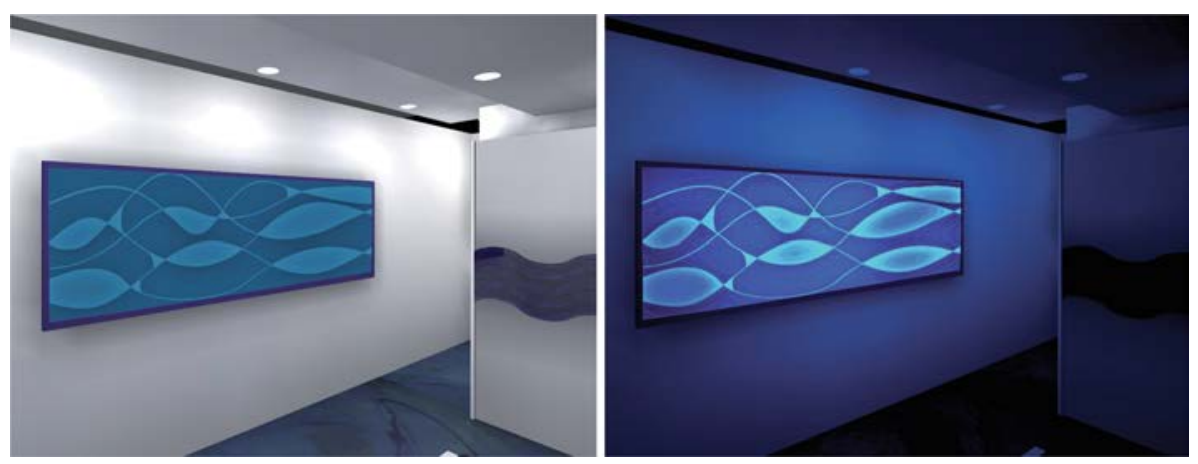

Fig. 1. Sleeping in the blue - insertion in interior of photoluminescent panel (Color figure online)

Richard Kelly, pioneer of qualitative lighting design, distinguished, already in the fifties, three fundamental functions of light:

- the light that provides general lighting for the environment AMBIENT LUMINESCENCE (see Fig. 2)

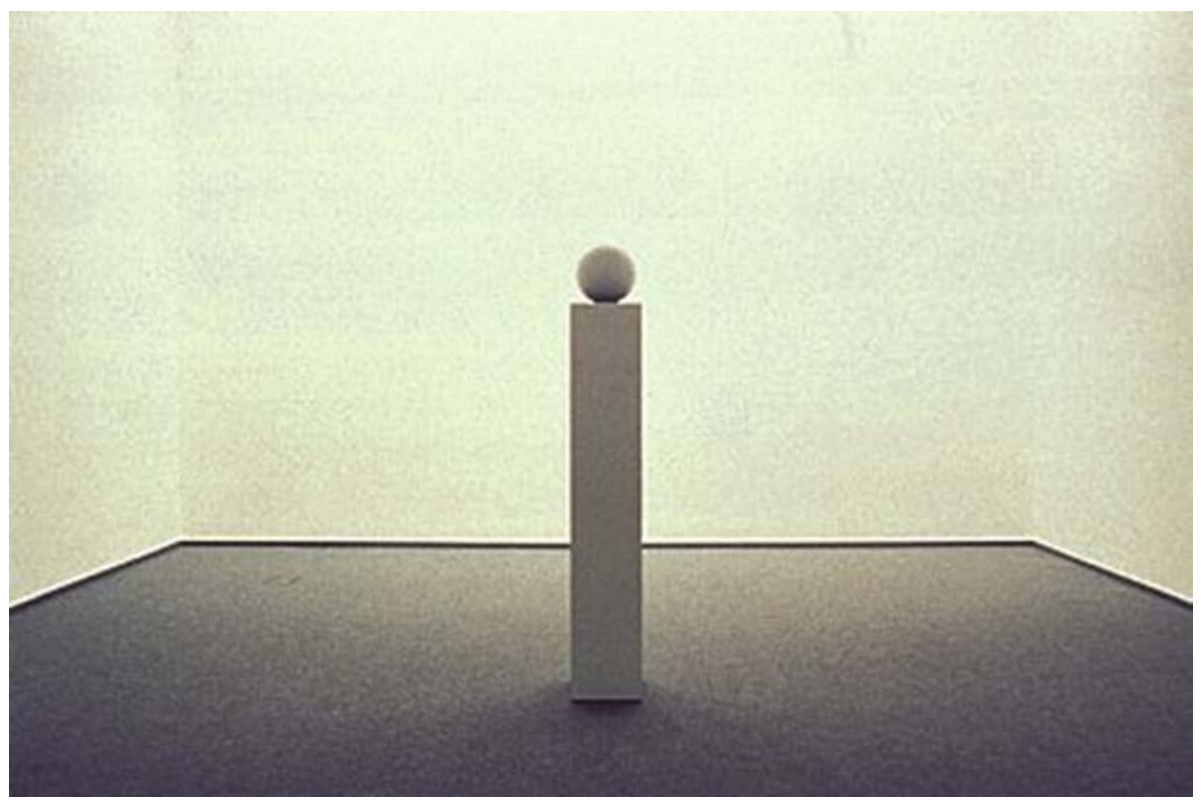

Fig. 2. Ambient luminescence - Richard Kelly

- the accent light that calls attention, separates and highlights - FOCAL GLOW (see Fig. 3) 


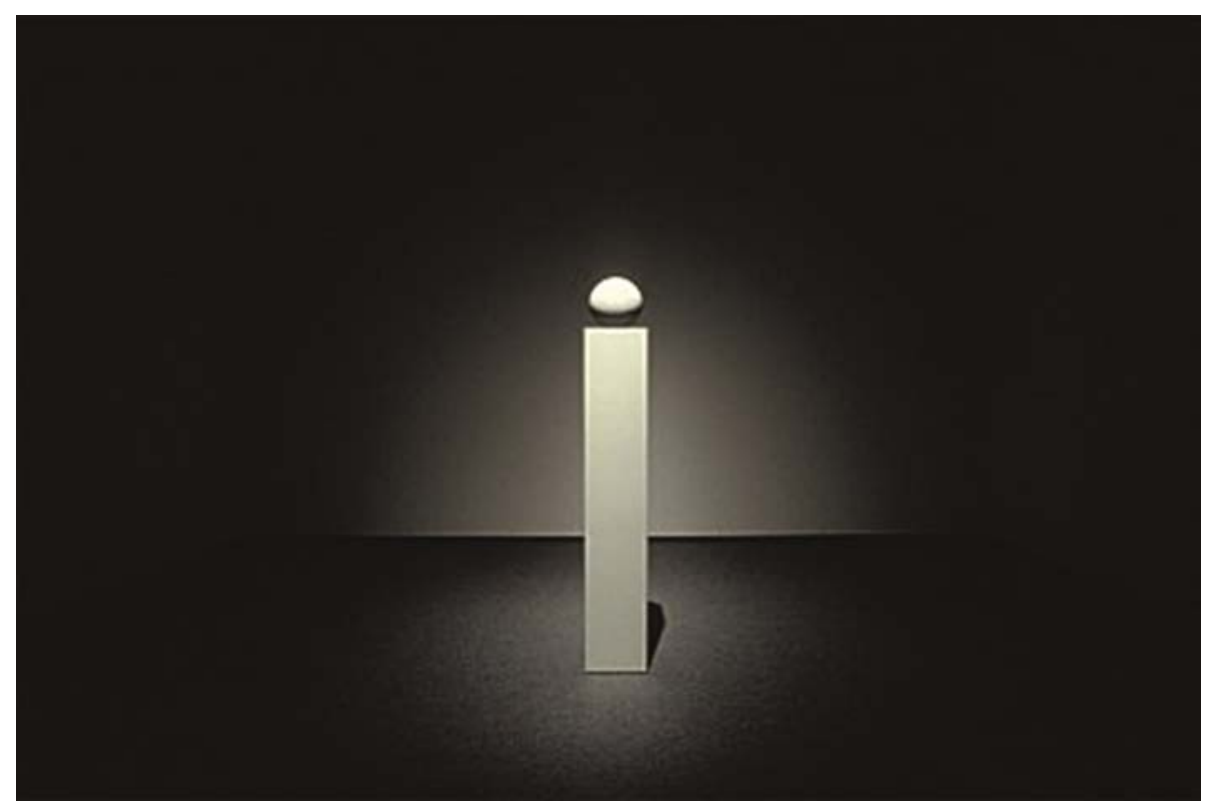

Fig. 3. Focal glow - Richard Kelly

- the light that captures, attracts and distracts, which becomes information for itself PLAY OF BRILLIANTS (see Fig. 4)

The lighting project is today more than then a combination of these three functions. These criteria for evaluating the light of the functions it can perform, applied in the various technical solutions available today, make it possible to design with extreme sensitivity the desired impact, effects and quality.

The luminous atmosphere that today can be designed has a lot of technology available to overcome the simple service of "making light" by being more sensitive to the emotional perception of the light effect.

Here in the organization of the position of the lights in addition to considering the right lighting the study of the position of the light points becomes the space-geometric location of the elements that unify the space. General lighting produces a light without shadows that minimizes shapes and volumes; reduces the importance of subjects and objects; suggests an idea of infinity, freedom, spatiality: the emotion of an experience of immersion in light. All this provokes a reassuring feeling in a welcoming and serene environment.

A diffused and uniform light does not mean an undifferentiated lighting for all the spaces and the contexts; on the contrary it involves the search for the appropriate solutions to the different needs that each specific environment requires according to the activities that must be carried out in it. The diffused light radiation, suitably manipulated, offers itself as a real construction material when it is used to configure spaces and volumes, dynamically varying the color and the refraction effects of the surfaces. 


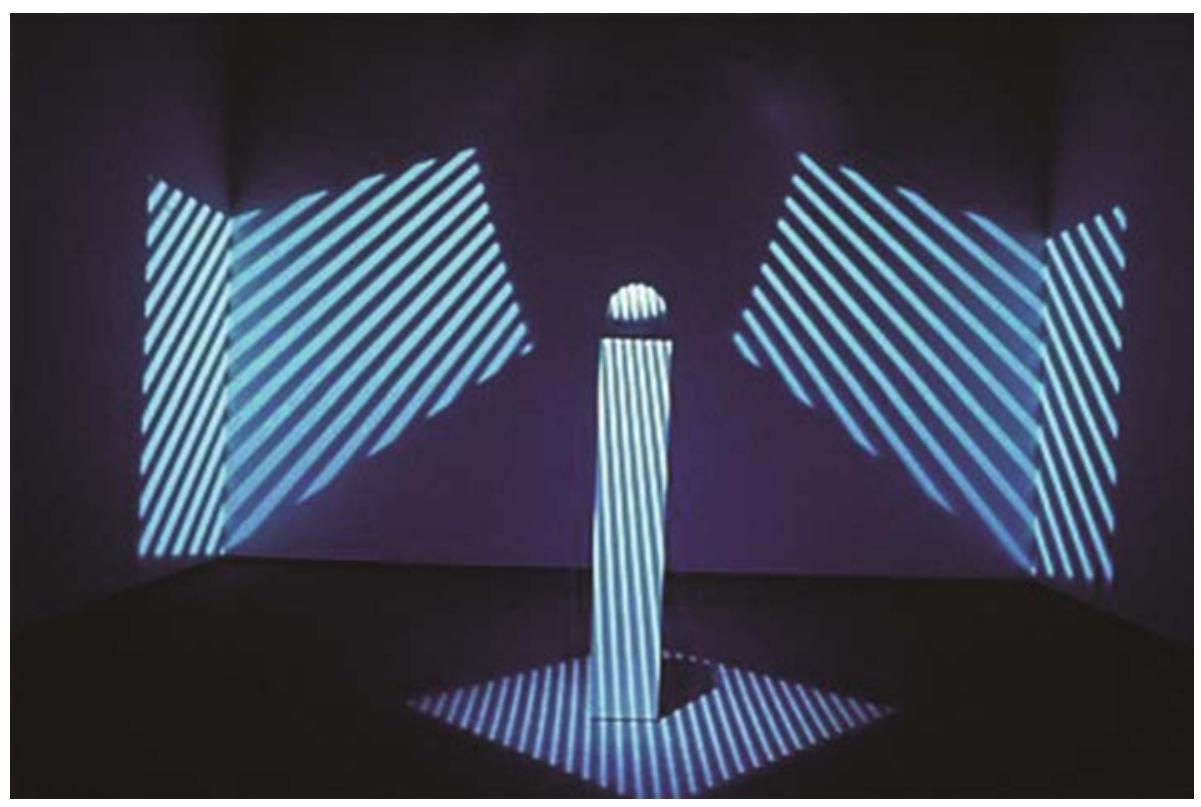

Fig. 4. Play of brilliants - Richard Kelly

We are now widely used to seeing environments that change color only with the temporary effect of artificial light. So we are culturally predisposed to accept and perceive, as a value, intangible light effects that can change the visual perception of the place. Since the 1960s, pop art has been able to accept advertising light as the most advanced expression of the lighting technology that modernity could have.

Las Vegas and Times Square have spread the culture of diffused light. The sixties were the years of fluorescent neon inaugurating "the era of the naked bulb", or the luminaire in sight.

The jump happened because of trying not to hide the light source anymore. Everything is left to the eye. The lamp becomes no longer just a source, but a legitimate medium in itself that characterizes the colors of the spaces.

The result is the diffusion of a fluid, magical, undulating weightless perception.

F. Wright writes in the "Testament" - "Glass tubes superimposed like bricks of a wall constitute the luminous surfaces" - we are in the age of "diaphanous" light.

With the advent of artificial light control we come to define a fourth dimension. To the three conventional ones we add the psychological and emotional one.

Says Vittorio Storaro "We must continue to learn the art of seeing. The eye must seize ... the whispers of the visible".

The availability that today gives us the photoluminescence is to educate us to an emotional perception. Doing little with the very imagination we need.

Lighting a garden for the whole night costs and wastes energy beyond the due. With photoluminescence it can become an intervention to interpret the amount of light useful and at the same time find new suggestions in the relationship with the environment through the light that is of "presence". 
A light that shows itself more than to show.

All this can also contribute to significant energy savings. In fact, thanks to the properties of the new pigments, photoluminescence can last for eight hours: one whole night. (see Figs. 5, 6 and 7).
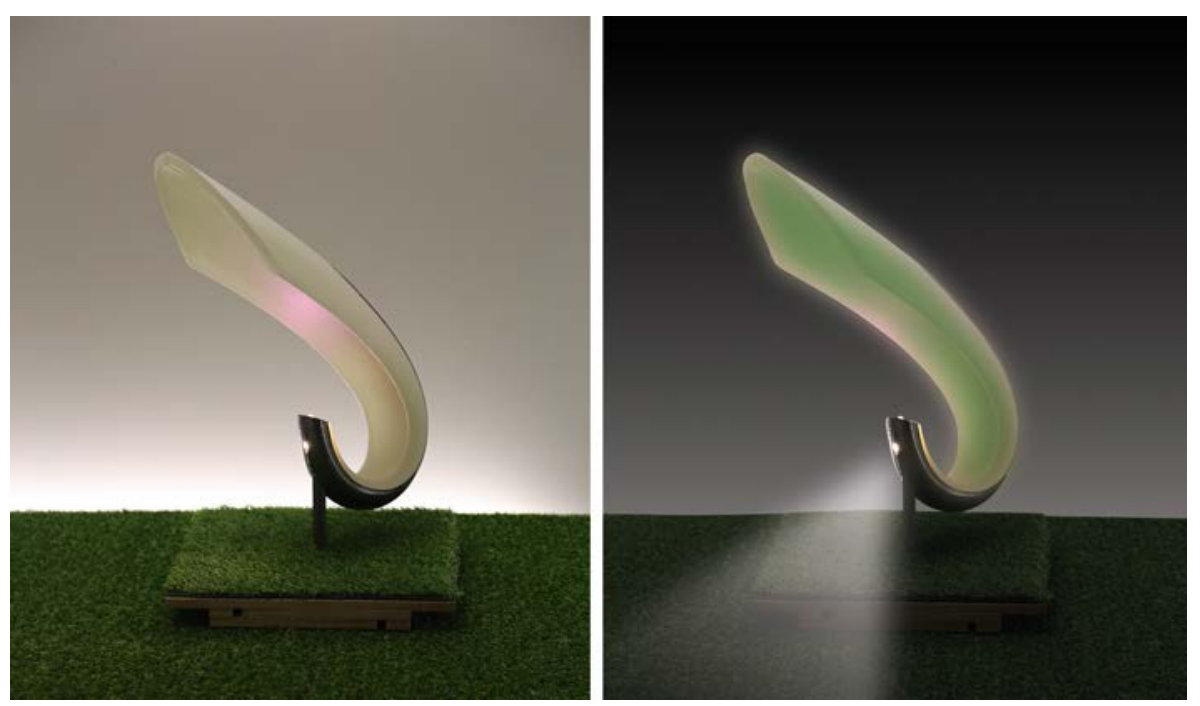

Fig. 5. Cochlea of M. Bottone and S. Toscano, project and experimental prototype of the Lighting Smart Lab, Dida - University of Florence
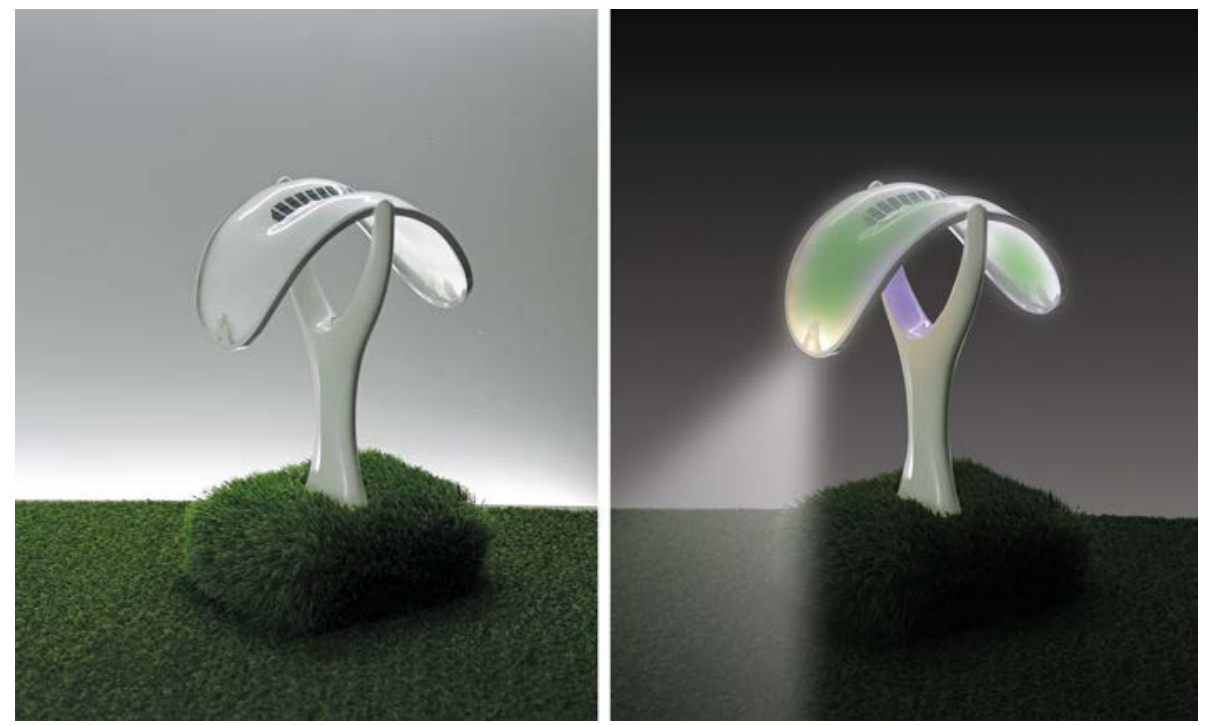

Fig. 6. PLDM of C. Buscemi, project and experimental prototype of the Lighting Smart Lab, Dida - University of Florence 

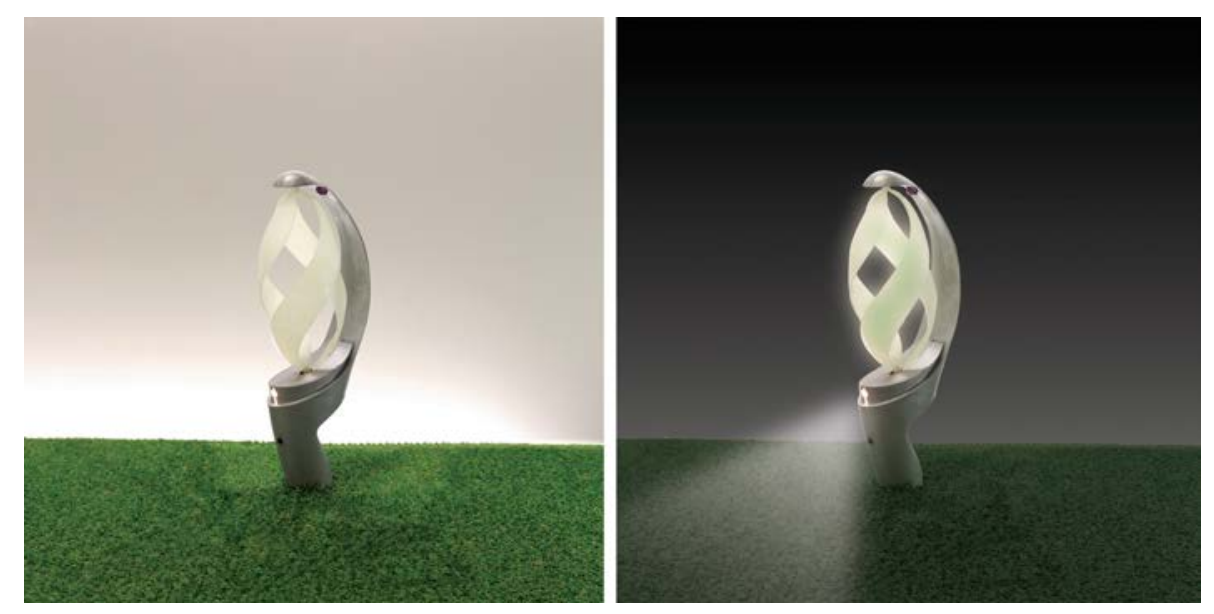

Fig. 7. Fleco of F. Parenti and S. Rinaldi, project and experimental prototype of the Lighting Smart Lab, Dida - University of Florence

The photoluminescence reinterprets the shadow light dichotomy, on/off, overcoming it by proposing a faint light, but useful to illuminate small spaces.

In front of it the eye is purified from the overload to which artificial light has accustomed it and, as in a moonless night of stars, slowly rediscovers the complexity of the world and new magical dimensions.

Luminescence thus becomes a friendly, comfortable form useful for regenerating psychological and environmental energies.

The new dimension of lighting design surpasses, with the support of increasingly sophisticated implementation tools, the commitment to make a correct contribution in lumens by expanding the lighting design towards a strongly cultural trajectory: educating to wellbeing by educating to the perception of light.

\section{References}

1. Alfarano G (2015) La luce che si fa vedere, Pietro Macchione Editore

2. Alfarano G (2016) L'abitare tra luce e buio, in Follesa S., sull'ABITARE, pp 134-143. Franco Angeli Editore

3. Alfarano G (2015) Forme di percezione della forma in Filieri J., Il prodotto da solo non basta, pp. 78-85 Altralinea Edizioni srl (2015)

4. Alfarano G (2016) La rapidità del design in Spennato A., Design e Prototipazione, pp 26-33, Editoriale Delfino

5. Arnheim R (1974) Il pensiero visivo. Einaudi, Torino

6. Celine L (1992) Viaggio al termine della notte. Corbaccio, Milano

7. Giannini AM, Marzi T, Viggiano MP (2011) Design. Percezione visiva e cognizione, psicologia dell'arte, la scelta del prodotto: emozioni, decisioni e neuroestetica, Giunti Editore

8. Kelly R, Neumann D (2010) The structure of light: Richard Kelly and the illumination of modern. Architecture Yale University Press, Connecticut 
9. Pierantoni R (1998) Verità a bassissima definizione. Einaudi, Torino

10. Rossi M (2008) Design della luce, Maggioli Editore

11. Tanizaki J (1992) Libro D'ombra. Bompiani, Milano

12. Van Hinte E, Bakker C (2000) Trespassers: Ispiration for eco-efficent design, Netherlands design istitute/010 pubblischera, Rotterdam 